\title{
Functional evaluation of human donation after cardiac death donor hearts using a continuous isolated myocardial perfusion technique: Potential for expansion of the cardiac donor population
}

\author{
Satoru Osaki, MD, PhD, Matthew R. Locher, PhD, Entela B. Lushaj, MD, PhD, Shahab A. Akhter, MD, and \\ Takushi Kohmoto, MD, PhD
}

\begin{abstract}
Objective: To investigate the resuscitation potential and contractile function in adult human donation after cardiac death (DCD) hearts by ex vivo perfusion.

Methods: With institutional review board approval and under the DCD protocol at the University of Wisconsin (UW) Organ Procurement Organization, 5 brain dead (BD) and 5 DCD donor hearts were evaluated. All BD hearts were declined for clinical transplantation because of coronary artery disease, advanced age, or social history. All hearts were preserved by flushing and cold storage with UW solution. By using our ex vivo perfusion system, the left ventricular end systolic pressure-volume relationship (LV-ESPVR) was assessed for 2 hours of oxygenated blood reperfusion.
\end{abstract}

\begin{abstract}
Results: All BD $(\mathrm{n}=5)$ and 4 DCD hearts were successfully resuscitated. One DCD heart was unable to be resuscitated due to prolonged warm ischemic time (WIT; 174 minutes). Mean WIT for resuscitated DCD hearts (from extubation to flushing with cold UW solution) was $34 \pm 3$ minutes (range, 26 to 40 minutes); mean cold ischemic time for BD donors was $211 \pm 31$ minutes compared with $177 \pm 64$ minutes for DCD donors. The calculated LV-ESPVRs for BD hearts after 1 and 2 hours of reperfusion were $6.9 \pm 0.7$ and $5.7 \pm 1.0 \mathrm{~mm}$ $\mathrm{Hg} / \mathrm{mL}$, respectively; LV-ESPVRs for DCD hearts after 1 and 2 hours of reperfusion were $5.6 \pm 1.5$ $(P=.45)$ and $3.0 \pm 0.7 \mathrm{~mm} \mathrm{Hg} / \mathrm{mL}(P=.07)$, respectively.
\end{abstract}

Conclusions: We successfully resuscitated and measured ex vivo cardiac function in human DCD and BD donor hearts. Resuscitation potential in DCD hearts was achieved when the WIT was less than 40 minutes. Contractile performance in DCD hearts tended to be lower compared with BD hearts. Further investigation with longer reperfusion periods seems warranted. (J Thorac Cardiovasc Surg 2014;148:1123-30)

Heart transplantation is an established and effective treatment for patients with end-stage heart failure; however, the shortage of donor organs is the most critical problem. The Organ Procurement and Transplantation Network (OPTN) reports indicate that the number of registrations on the heart waiting list is approximately 3000 in a recent 3 -year period and $15 \%$ of patients die within 1 year while awaiting a donor heart (http://optn.transplant.hrsa.gov/). To maintain or increase the present transplantation rate, donation after cardiac death (DCD) donors have been proposed as another donor source. The concept of heart

\footnotetext{
From the Division of Cardiothoracic Surgery, Department of Surgery, University of Wisconsin School of Medicine and Public Health, Madison, Wis.

This research was supported by University of Wisconsin Institute for Clinical and Translational Research Grant (233-PRJ49YZ).

Disclosures: Authors have nothing to disclose with regard to commercial support.

Read at the 94th Annual Meeting of The American Association for Thoracic Surgery, Toronto, Ontario, Canada, April 26-30, 2014.

Satoru Osaki and Matthew R. Locher contributed equally to this work.

Received for publication April 8, 2014; revisions received June 19, 2014; accepted for publication June 27, 2014

Address for reprints: Satoru Osaki, MD, PhD, 600 Highland Ave, H4/337 Clinical Science Center, Madison, WI 53792-3236 (E-mail: osaki@surgery.wisc.edu).

$0022-5223 / \$ 36.00$

Copyright (C) 2014 by The American Association for Thoracic Surgery

http://dx.doi.org/10.1016/j.jtcvs.2014.06.050
}

transplantation using DCD donors existed in the 1960s before brain death became legally accepted and the first successful clinical human heart transplantation in an adult was performed using a DCD heart allograft in 1967 by Christiaan Barnard. ${ }^{1}$ In the current era, Boucek and collegues ${ }^{2}$ reported the short-term results of 3 infants undergoing successful orthotopic heart transplantation from DCD donors. For noncardiac organ transplantation, clinical studies from several institutions show that DCD donors are reliable donor sources for organs such as kidney ${ }^{3}$ and liver. ${ }^{4}$ The OPTN data show a progressive increase in the rate of organ recovery from DCD donors (1089 DCDs in 2012, compared with 189 in 2002) and that these donors accounted for $12 \%$ of all deceased donors in 2012 (http://optn.transplant.hrsa.gov/). In particular, at several Organ Procurement Organization programs, DCD donors accounted for more than $20 \%$ of all deceased donors. Although the use of DCD donors for noncardiac organ transplant has been increasing, the potential for heart transplantation from DCD donors remains unrealized, because of potentially severe myocardial damage due to unavoidable warm ischemia (from the withdrawal of life support to flushing with cold preservative solution).

According to the 1995 Maastricht categories, ${ }^{5}$ DCD donors are classified as uncontrolled or controlled donors. 


$\begin{array}{ll}\text { Abbreviations and Acronyms } \\ \text { BD } & =\text { brain dead } \\ \text { CIT } & =\text { cold ischemic time } \\ \text { DCD } & =\text { donation after cardiac death } \\ \text { LV-EDP } & \text { left ventricular end diastolic } \\ & \text { pressure } \\ \text { LV-ESP } & \text { left ventricular end systolic } \\ & \text { pressure } \\ \text { LV-EDPVR }= & \text { left ventricular end diastolic } \\ & \text { pressure-volume relationship } \\ \text { LV-ESPVR }= & \text { left ventricular end systolic } \\ & \text { pressure-volume relationship } \\ \text { LV dP/dt max }= & \text { left ventricular maximum of first } \\ & \text { derivative of pressure } \\ \text { LV dP/dt min }= & \text { left ventricular minimum of first } \\ & \text { derivative of pressure } \\ \text { LVP } & =\text { left ventricular pressure } \\ \text { OPTN } & \text { Organ Procurement and } \\ & \text { Transplantation Network } \\ \text { UW } & =\text { University of Wisconsin } \\ \text { UWHC-OPO = } & \text { University of Wisconsin Hospital } \\ & \text { and Clinics-Organ Procurement } \\ & \text { Organization } \\ \text { WIT } & \text { warm ischemic time } \\ & \end{array}$

Uncontrolled donors are dead on arrival (category I) or had an unsuccessful resuscitation (category II). Controlled donors have an awaited cardiac arrest (category III) or develop cardiac arrest while brain dead (category IV). The so-called Maastricht category III, withdrawal of donor treatment (usually in the intensive care unit or operating room), is a controlled DCD technique and the only one presently in use in the United States, including our institution. This category III DCD donor could potentially be used for clinical heart transplantation.

Over the past decades, many animal experiments have been undertaken to evaluate the feasibility of heart transplantation from DCD donors, using various reperfusion strategies and animal DCD models. Some researchers indicated excellent results in animal DCD heart transplant experiments. Shirakura and colleagues ${ }^{6}$ reported satisfactory functional recovery of canine hearts after a 24-hour period of preservation in a 30-minute warm ischemic DCD transplant model, and Gundry and colleagues ${ }^{7}$ achieved long-term survival of baboons receiving transplantation of hearts harvested from 30-minute warm ischemic DCD donors. However, these successful experimental outcomes are largely attributable to the application of multiple pretreatments, which, in humans, would be ethically unacceptable. In our previous animal studies, ${ }^{8,9}$ we evaluated acute posttransplant graft function using a 30-minute warm ischemic pig DCD model without any cardioprotective pretreatments except for heparin, and this graft functional recovery rate was approximately $80 \%$ of the pretransplant (normal) value. These animal studies support the potential acceptability of 30-minute warm ischemic DCD cardiac grafts. However, the acceptability of warm ischemic time (WIT) for human adult DCD hearts is still unknown because resuscitation potential and functional recovery after warm ischemia have not yet been elucidated.

The purpose of this study was to evaluate the resuscitation potential and contractile function in adult human DCD hearts using an ex vivo perfusion system and investigate functional recovery for human DCD hearts compared with human brain dead (BD) hearts.

\section{MATERIALS AND METHODS DCD and BD Donors}

Five BD and 5 DCD donor hearts were evaluated in this study, with institutional review board approval and using the deceased donor (BD and DCD) protocol in the University of Wisconsin Hospital and Clinics-Organ Procurement Organization (UWHC-OPO). ${ }^{10}$

All DCD donor hearts were obtained from donors eligible for other organ transplant protocols in the UWHC-OPO, who agreed to donate the heart for research purposes. Each organ was checked separately on the consent form. All DCD donors in this study were brought to the operating room before the withdrawal of life support. While the patient was fully supported, 30,000 units of heparin were given intravenously to facilitate subsequent organ flushing. The patient's physician of record withdrew life support by stopping intravenous medications and extubation. The organ recovery procedure commenced only after an additional 5 minutes elapsed after the declaration of death, as described in the 1997 Institute of Medicine Guidelines. $^{11}$ Five minutes after the declaration of death, median sternotomy and a midline abdominal incision were made and the inferior vena cava was dissected immediately to decompress the heart. All DCD hearts were asystolic when they were explanted. After organ recovery for clinical transplantation, the ascending aorta was cannulated and approximately $2 \mathrm{~L}$ of UW solution was infused in situ. Then the heart was removed en bloc, stored in UW solution at $4^{\circ} \mathrm{C}$, and transported to our research laboratory.

All BD hearts were obtained from donors who were ineligible for our $\mathrm{BD}$ heart transplant protocol, and who agreed to donate the heart for research purposes. Each organ was checked separately on the consent form. All BD hearts were preserved using our institutional method of BD heart recovery. After administration of 30,000 units of heparin, the ascending aorta was clamped and approximately 2 L of UW solution was infused through the aortic cannula. The inferior vena cava was divided immediately to decompress the heart. The heart was removed en bloc and stored in UW solution at $4^{\circ} \mathrm{C}$.

WIT was defined as the interval between withdrawal of life support and cold flush of UW solution; cold ischemic time (CIT) was defined as the interval between cold flush and oxygenated blood reperfusion of the graft.

\section{Ex Vivo Myocardial Perfusion System}

The perfusion circuit was an open system and the reperfusion protocol was similar to that previously described (Figure 1). ${ }^{9}$ The system consisted of a Terumo pediatric membrane oxygenator with the reservoir containing a filter (Terumo Cardiovascular Systems, Ann Arbor, Mich), a circulating water bath, a hemofilter, a roller head pump, a temperature meter, a leukocyte-depletion filter (Pall, Glen Cove, NY), and 6.4-mm (0.25-inch) connective tubing. A new circuit was used for each procedure in a semisterile manner. The total circuit volume was $600 \mathrm{~mL}$. Coronary venous 


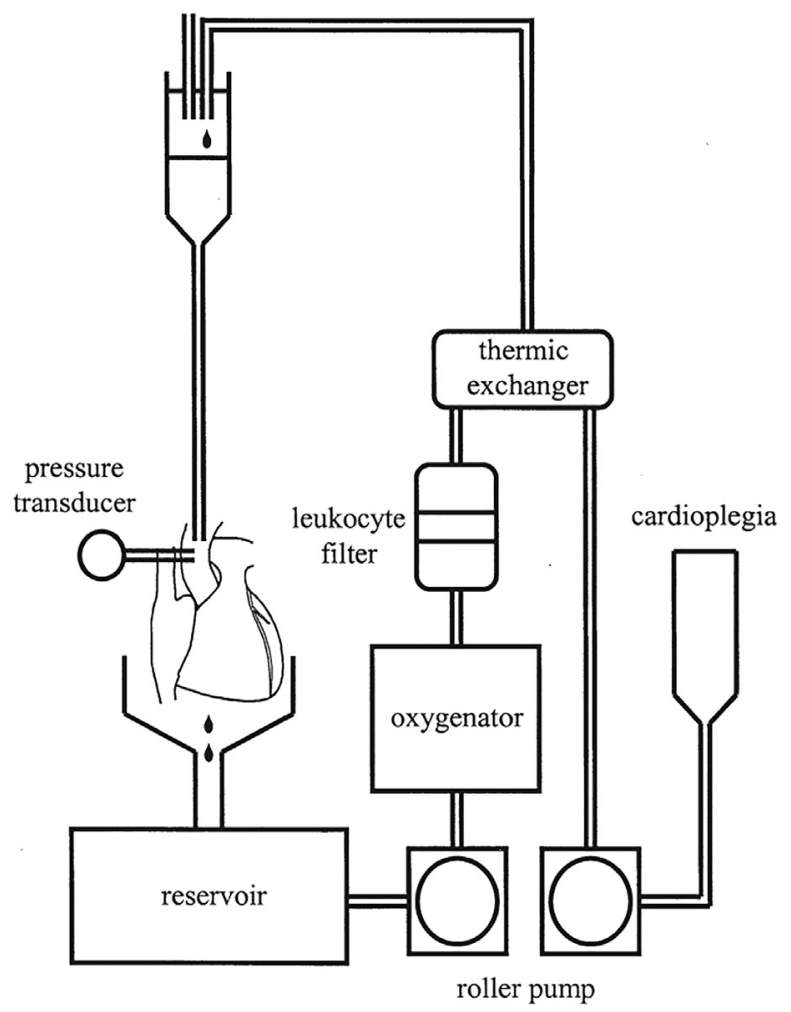

FIGURE 1. Ex vivo perfusion system. ${ }^{9}$

blood drained spontaneously and thebesian flow drained through stab wounds in the right and left ventricles, all of which was collected in a large funnel and directed to the reservoir where it was warmed by an internal heating element via the water bath. The blood was then drawn from the reservoir by the roller head pump and passed through the hemofilter on its return to the membrane oxygenator. This oxygenated blood was passed through the leukocyte-depletion filter and pumped to the upper small blood reservoir. Then leukocyte-depleted oxygenated blood was perfused through the coronary arteries by gravity. This circuit was primed with 4 units of recently outdated, type-specific, and noncrossmatched packed human red blood cells and crystalloid, such as lactated Ringer and 5\% dextrose solutions. The human red blood cells were washed to remove excess potassium and citrate by mixing with $1000 \mathrm{~mL}$ of normal saline solution. Two hundred units of heparin and $300 \mathrm{mg}$ of calcium gluconate were added to each unit of packed cells. An additional 1000 units of heparin were added to the circuit. The prime was recirculated through the system via a heated water bath and the perfusate reached $20^{\circ} \mathrm{C}$. Before the heart was reperfused, corrective measures were required to adjust the sodium concentration, chloride concentration, potassium concentration, $\mathrm{pH}$, and calcium concentration of the perfusate to make the solution physiologic, and these values were equivalent between the groups. The perfusate was oxygenated through the membrane oxygenator with an admixture of compressed air and carbogen $\left(5 \% \mathrm{CO}_{2}, 21 \% \mathrm{O}_{2}\right.$, and balance $\left.\mathrm{N}_{2}\right)$. The percentage of carbogen was adjusted to maintain a carbon dioxide tension of 30 to $34 \mathrm{~mm} \mathrm{Hg}$. The hemoglobin oxygen saturation was $100 \%$ throughout the experiment as determined by periodic blood gas determinations.

For reperfusion, after the hearts were transported to our laboratory, a custom aortic cannula was connected to the aortic root. For the controlled initial perfusion, 4:1 leukocyte-depleted blood cardioplegia was infused for 20 minutes at $20^{\circ} \mathrm{C}$ with a perfusion pressure of $40 \mathrm{~mm} \mathrm{Hg}$. The first solution that passed through the heart was discarded into a vacuum bucket. After the initial reperfusion, myocardial perfusion was then resumed with leukocyte-depleted oxygenated blood at $20^{\circ} \mathrm{C}$ with a perfusion pressure of
TABLE 1. Donor characteristics

\begin{tabular}{|c|c|c|c|c|c|c|}
\hline $\begin{array}{l}\text { Type of } \\
\text { donor }\end{array}$ & $\begin{array}{c}\text { Age } \\
(\mathbf{y})\end{array}$ & Gender & $\begin{array}{c}\text { Cause of } \\
\text { brain death } \\
\text { or major } \\
\text { brain injury }\end{array}$ & $\begin{array}{l}\text { WIT } \\
(\mathrm{min})\end{array}$ & $\begin{array}{c}\text { CIT } \\
(\mathbf{m i n})\end{array}$ & Resuscitation \\
\hline BD 1 & 54 & Female & Anoxia & 0 & 115 & Yes \\
\hline BD 2 & 50 & Female & Anoxia & 0 & 293 & Yes \\
\hline BD 3 & 21 & Female & Anoxia & 0 & 251 & Yes \\
\hline BD 4 & 59 & Male & ICH & 0 & 178 & Yes \\
\hline BD 5 & 66 & Male & Stroke & 0 & 220 & Yes \\
\hline DCD 1 & 31 & Female & Head trauma & 174 & 53 & No \\
\hline DCD 2 & 36 & Female & Stroke & 40 & 77 & Yes \\
\hline DCD 3 & 58 & Female & ICH & 32 & 349 & Yes \\
\hline DCD 4 & 56 & Male & Head trauma & 26 & 83 & Yes \\
\hline DCD 5 & 55 & Female & $\mathrm{ICH}$ & 36 & 198 & Yes \\
\hline
\end{tabular}

WIT, Warm ischemic time (from withdrawal of life support to cold flush of University of Wisconsin solution); CIT, cold ischemic time (from cold flush to oxygenated blood reperfusion); $B D$, brain dead; $D C D$, donation after cardiac death; $I C H$, intracranial hemorrhage.

$40 \mathrm{~mm} \mathrm{Hg}$. The perfusion pressure was increased to $80 \mathrm{~mm} \mathrm{Hg}$ and the temperature was increased stepwise to $37^{\circ} \mathrm{C}$ over the next 30 minutes. All blood parameters were monitored throughout the experiment by blood gas examination, and were corrected to be within physiologic range. If the hearts were fibrillating while rewarming, electric cardioversion with ventricular pacing was used as needed.

\section{Measurement of Contractile Function}

Isovolumetric left ventricular pressure (LVP) was measured for 2 hours with a transducer connected to a thin, saline-filled latex balloon inserted into the left ventricle through the mitral valve from an incision in the left atrium. Balloon volume was adjusted initially to a diastolic LVP of $0 \mathrm{~mm} \mathrm{Hg}$ so that any subsequent increase in diastolic LVP reflected an increase in left ventricle wall stiffness (ie, diastolic contracture). Left ventricular end systolic pressure-volume relationship (LV-ESPVR) and left ventricular end diastolic pressure-volume relationship (LV-EDPVR) were calculated from pressure-volume relation curves obtained by changing the balloon volume. Left ventricle maximum and minimum of the first derivative of pressure $(\mathrm{dP} / \mathrm{dt} \max$ and $\mathrm{dP} / \mathrm{dt} \min$, respectively) were obtained from a pressure curve acquired from a high-fidelity micromanometer catheter (Miller Instruments, Houston, Tex). Left ventricular systolic (LV-ESPVR and $\mathrm{dP} / \mathrm{dt} \max$ ) and left ventricular diastolic (LV-EDPVR and $\mathrm{dP} / \mathrm{dt} \min$ ) functions were analyzed.

\section{Statistical Analysis}

Statistical significance of any difference between the BD and DCD hearts was determined with an independent sample $t$ test. The results are given as the mean \pm standard error. All analyses were performed using the SPSS for Windows version 22.0 (SPSS Inc, Chicago, Ill).

\section{RESULTS}

\section{Donor Characteristics}

The donor characteristics are shown in Table 1 . The mean age of all donors was $49 \pm 14$ years old. The mean age was not different between the groups (BD vs DCD; $50 \pm 8$ vs 47 \pm 6 years; $P=.78$ ). Seven donors were female ( $3 \mathrm{BD}$ and 4 DCD donors). Causes of brain death or major brain injury were anoxia for 3 DCDs, intracranial hemorrhage for 1 $\mathrm{BD}$ and 2 DCDs, stroke for $1 \mathrm{BD}$ and $1 \mathrm{DCD}$, and head 
TABLE 2. Contractile function for BD and DCD hearts

\begin{tabular}{lccc}
\hline & 60 min after reperfusion & 90 min after reperfusion & 120 min after reperfusion \\
\hline $\mathrm{dP} / \mathrm{dt}$ max for BDs $(\mathrm{mm} \mathrm{Hg} / \mathrm{s})$ & $983 \pm 102$ & $969 \pm 77.6$ & $801 \pm 112$ \\
$\mathrm{dP} / \mathrm{dt}$ max for DCDs $(\mathrm{mm} \mathrm{Hg} / \mathrm{s})$ & $781 \pm 270$ & $734 \pm 180$ & $481 \pm 161$ \\
$P$ value & .56 & .30 & .16 \\
$\mathrm{LV}-\mathrm{ESPVR}$ for BDs $(\mathrm{mm} \mathrm{Hg} / \mathrm{mL})$ & $6.9 \pm 0.7$ & $6.4 \pm 0.9$ & $5.7 \pm 1.0$ \\
$\mathrm{LV}-\mathrm{ESVPR}$ for DCDs $(\mathrm{mm} \mathrm{Hg} / \mathrm{mL})$ & $5.6 \pm 1.5$ & $5.0 \pm 1.1$ & $3.0 \pm 0.7$ \\
$P$ value & .45 & .36 & .07 \\
$\mathrm{dP} / \mathrm{dt}$ min for BDs $(\mathrm{mm} \mathrm{Hg} / \mathrm{s})$ & $576 \pm 48.1$ & $494 \pm 63.3$ & $511 \pm 71.8$ \\
$\mathrm{dP} / \mathrm{dt}$ min for DCDs $(\mathrm{mm} \mathrm{Hg} / \mathrm{s})$ & $507 \pm 130$ & .62 & $332 \pm 93.1$ \\
$P$ value & $-0.69 \pm 0.43$ & $-0.69 \pm 0.34$ & .18 \\
$\mathrm{LV}-\mathrm{EDPVR}$ for BDs $(\mathrm{mm} \mathrm{Hg} / \mathrm{mL})$ & $-0.70 \pm 0.49$ & $-0.71 \pm 0.34$ & $-0.82 \pm 0.62$ \\
$\mathrm{LV}-\mathrm{EDPVR}$ for DCDs $(\mathrm{mm} \mathrm{Hg} / \mathrm{mL})$ & .98 & .96 & $-0.11 \pm 0.50$ \\
$P$ value & .41 & .41 \\
\hline
\end{tabular}

$\overline{B D}$, Brain dead donor; $D C D$, donation after cardiac death donor; $d P / d t$ max, left ventricular maximum of first derivative of pressure; $L V$ - $E S P V R$, left ventricular end systolic pressure-volume relationship; $d P / d t$ min, left ventricular minimum of first derivative of pressure; $L V$ - $E D P V R$, left ventricular end diastolic pressure-volume relationship.

trauma for 2 DCDs. Heparin was administered to all DCD donors before withdrawal of support. The mean WIT for the 4 DCD hearts that were resuscitated by ex vivo perfusion was $34 \pm 3$ minutes (range, 26-40 minutes). The mean CIT for BD donors was $211 \pm 31$ minutes. The mean CIT for the 4 resuscitated DCD hearts was $177 \pm 64$ minutes, which was similar to that of BD hearts $(P=.65)$. Reasons for declining clinical heart transplantation for BD donors were significant coronary artery disease diagnosed by coronary angiogram for 3 donors, advanced age for 1 donor, and complicated social history for 1 donor.

\section{Resuscitation Potential and Contractile Function}

Five BD (100\%) and 4 DCD $(80 \%)$ hearts were successfully resuscitated and evaluated for contractile function. However, 1 DCD heart with 174 minutes of WIT did not start to beat after oxygenated blood reperfusion.

Contractile function is shown in Table 2 and Figures 2 through 4. Left ventricular systolic functions $(\mathrm{dP} / \mathrm{dt} \max$ and LV-ESPVR) for DCD hearts were comparable with those of BD hearts at 60 and 90 minutes after reperfusion $(\mathrm{dP} / \mathrm{dt} \max , P=.56$ at 60 minutes and $P=.30$ at $90 \mathrm{mi}-$ nutes; LV-ESPVR, $P=.45$ at 60 minutes and $P=.36$ at 90 minutes), however, the values for DCD hearts at 120 minutes after reperfusion tended to be lower compared with BD donors (dP/dt max, $P=.16$; LV-ESPVR, $P=.07)$. Left ventricular diastolic functions $(\mathrm{dP} / \mathrm{dt} \min$ and LV-EDPVR) for DCD hearts were comparable with those of BD hearts at 60 and 90 minutes after reperfusion (dP/dt min, $P=.67$ at 60 minutes and $P=.62$ at 90 minutes; LV-EDPVR, $P=.98$ at 60 minutes and $P=.96$ at 90 minutes), however, the values for DCD hearts at 120 minutes after reperfusion tended to be lower compared with $\mathrm{BD}$ donors, especially for $\mathrm{dP} / \mathrm{dt} \min (P=.18)$.

\section{DISCUSSION}

This study is the first report to evaluate resuscitation potential and ex vivo nonworking contractile myocardial function in acute phase for human DCD hearts compared with human discarded BD hearts. Ten deceased donor hearts were evaluated in this study and 9 hearts including 5 BD and 4 DCDs within 40 minutes of WIT $(90 \%)$ were successfully resuscitated using our custom ex vivo isolated myocardial perfusion system. Our study has shown that ex vivo contractile function for 90 minutes of reperfusion in the resuscitated human DCD hearts was comparable with that of human BD hearts. However, after 2 hours of reperfusion, the graft function for DCD donors tended to deteriorate compared with BD hearts.

The major cause of DCD graft deterioration is warm ischemic injury. Warm ischemia, commonly defined as the interval of time between extubation (as the definitive withdrawal of treatment) and the initiation of cold perfusion for organ preservation, is unavoidable during the process of donation after cardiac death or circulatory arrest. ${ }^{12,13}$ This is the most critical concern for DCD allografts, because it is not observed in the hearts of $\mathrm{BD}$ donors and causes more severe graft injury than cold ischemia. The exposure to warm ischemia leads to a significant deletion of myocardial energy stores, anaerobic metabolism, and development of intracellular acidosis, ${ }^{14}$ which eventually causes myocyte death after reperfusion. ${ }^{15}$ Moreover, DCD hearts would be forced to attempt to support an entire circulatory load while functioning in an increasingly hypoxic environment during the period from extubation to cardiac arrest, ${ }^{16}$ whereas BD cardiac allografts do not suffer such load impairments induced by hypoxia because cardiac arrest is initiated by using cardioplegic solution and venous drainage. In our previous animal study, we clearly documented the changes in pressure and volume loads during the increasing hypoxia in the DCD animal model; the peak distending pressure was about $150 \%$ of baseline and the peak ventricular distended volume was $132 \%$ of baseline. ${ }^{17}$ These unfavorable and unavoidable injuries in the DCD context can greatly affect the DCD cardiac graft function. 


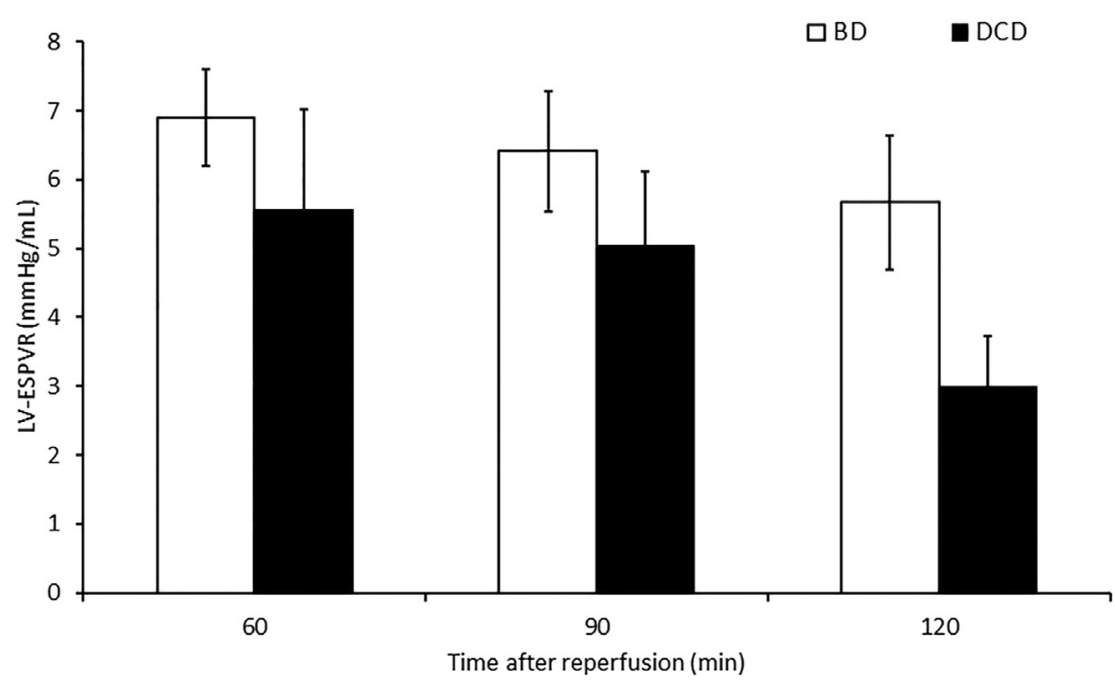

FIGURE 2. Contractile function for BD and DCD donors. $B D$, Brain dead; $D C D$, donation after cardiac death; $L V$-ESPVR, left ventricular end systolic pressure-volume relationship.

Several animal DCD heart transplant studies have been conducted to elucidate the feasibility of DCD cardiac allografts under these unfavorable conditions, and to evaluate their functional recovery after transplant and the acceptable limits of WIT. ${ }^{6-9}$ Although several species such as pig, ${ }^{8,9}$ dog, ${ }^{6}$ and baboon ${ }^{7}$ were used and various strategies of reperfusion and organ preservation were applied to these studies, the results support the potential acceptability of up to 30 minutes of WIT. Gundry and colleagues $^{7}$ achieved long-term survival of baboons receiving transplantation of hearts harvested from DCD donors within 30 minutes of WIT. However, at the structural
A

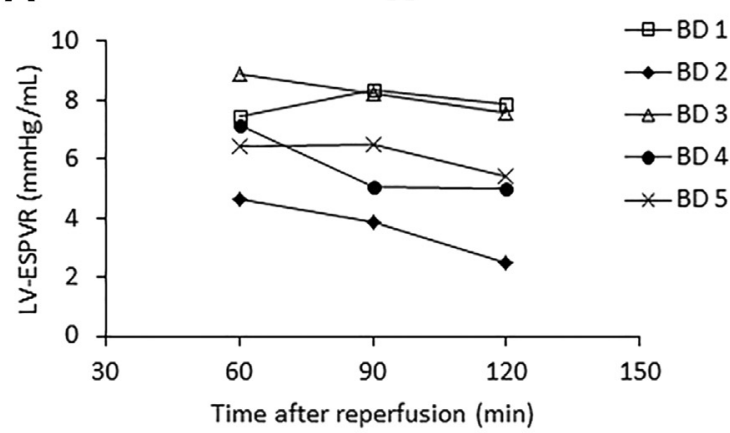

C

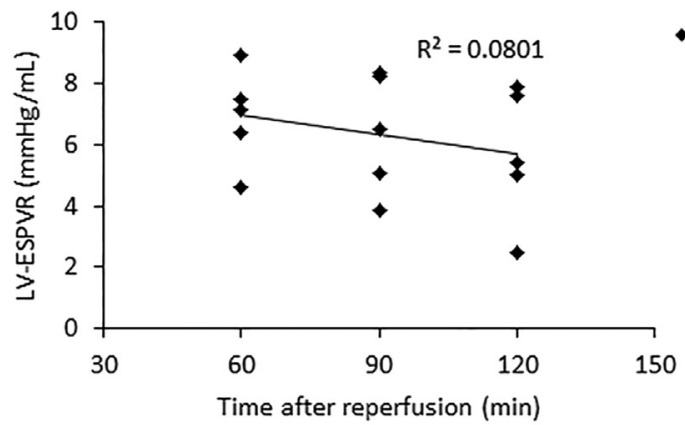

B

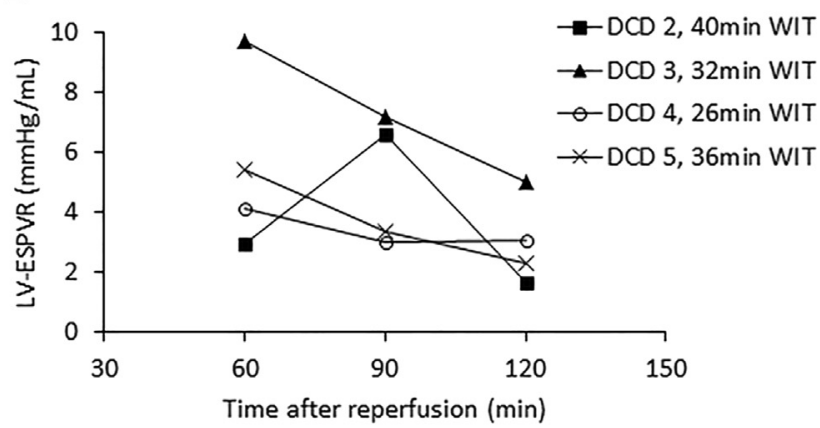

$\mathrm{DCD}$, trendline

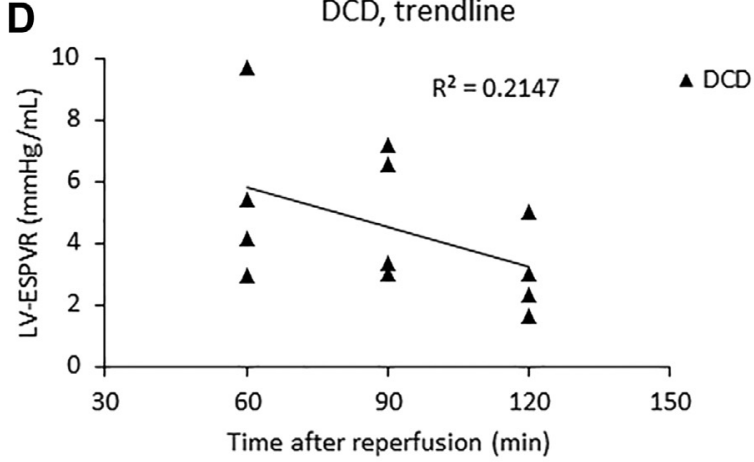

FIGURE 3. Individual comparison and trend line of LV-ESPVR in BD and DCD hearts. A, Individual comparison of LV-ESPVR for BD hearts. $\mathrm{B}$, Individual comparison of LV-ESPVR for DCD hearts. C, Trend line of LV-ESPVR for BD hearts. D, Trend line of LV-ESPVR for DCD hearts. BD, Brain dead; $D C D$, donation after cardiac death; $L V$-ESPVR, left ventricular end systolic pressure-volume relationship; WIT, warm ischemic time. 
A

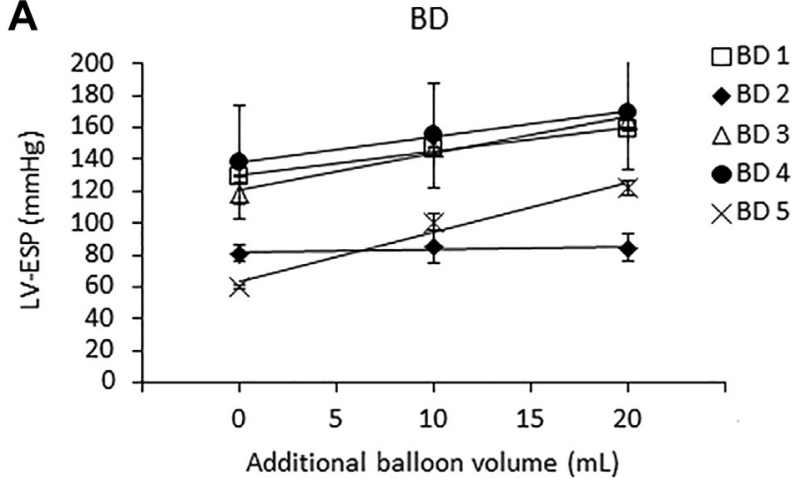

C

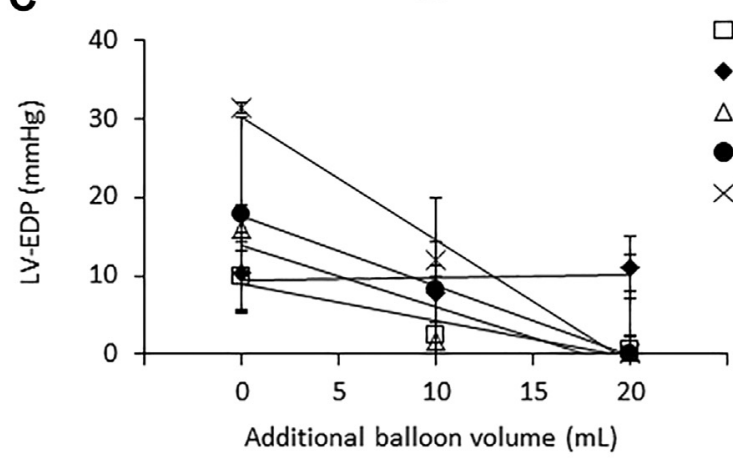

B

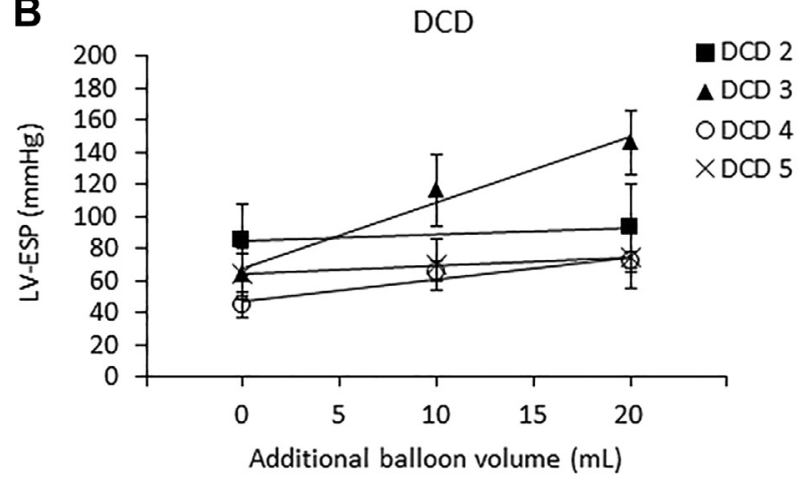

D

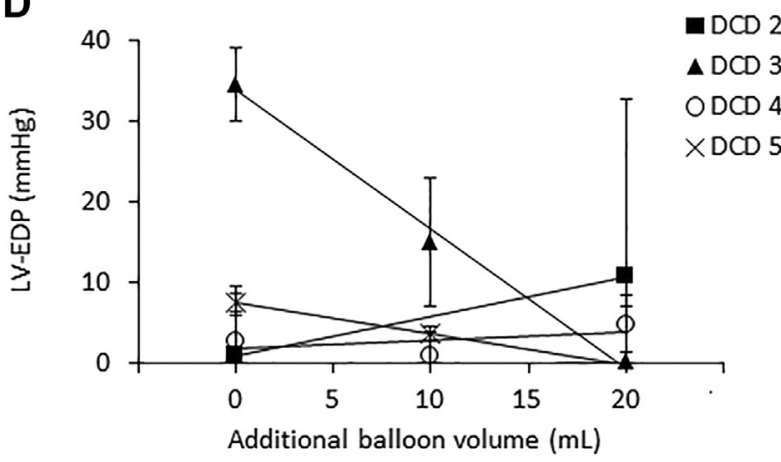

FIGURE 4. Individual left ventricular end systolic pressure-volume relationship (LV-ESPVR) and left ventricular end diastolic pressure-volume relationship (LV-EDPVR) plots in BD and DCD hearts at 90 minutes of reperfusion. A, Individual LV-ESPVR plots for BD hearts. B, Individual LV-ESPVR plots for DCD hearts. C, Individual LV-EDPVR plots for BD hearts. D, Individual LV-EDPVR plots for DCD hearts. $L V$-ESP, Left ventricular end systolic pressure; $B D$, brain dead; $D C D$, donation after cardiac death; $L V-E D P$, left ventricular end diastolic pressure.

level, global warm ischemia of 20 minutes can result in irreversible ischemic injury (ie, isolated cell death or tiny islands of cell death). ${ }^{18}$

To date, the clinical experience of human DCD heart transplantation has been limited and only a few case reports have been published. The first 2 adult human heart transplantations performed by Christiaan Barnard in $1967^{1}$ and $1968^{19}$ were from DCD donors because there was no formal definition of brain death at that time. Both recipients showed remarkable recovery and reversibility of heart failure symptoms after transplant, however, the first patient died as a result of pneumonia on postoperative day 18 and the second patient survived for 18 months before dying from chronic rejection. There was no detailed information on the interval from withdrawal of care to cardiac arrest for these 2 DCD donors, except the time from certification of death to initiation of median sternotomy was 5 minutes. The first pediatric human DCD heart transplantation was reported by Boucek and colleagues ${ }^{2}$ in 2008. According to this report, the mean WIT was 18.3 minutes (11.5, 16.0, and 27.5 minutes, respectively) and the 6-month survival was $100 \%$ for the 3 DCD heart transplant recipients, compared with $84 \%$ survival for 17 control infants who received transplants procured through standard organ donation. These DCD heart transplant recipients have had functional and immunologic outcomes similar to those of controls. A possible greater tolerance for global warm ischemia of the immature myocardium needs to be considered compared with the adult heart because of physiologic features in immature hearts. ${ }^{20}$

Recently, 2 case reports regarding postresuscitated functional evaluation for human DCD hearts have been published. ${ }^{21,22}$ Ali and colleagues ${ }^{21}$ reported biventricular cardiac functional recovery in a human DCD donor resuscitated by using an extracorporeal perfusion after cardiac death. The donor was a 57-year-old woman with irreversible neurologic damage from intracranial hemorrhage. The WIT from extubation to initiation of extracorporeal perfusion was 24 minutes and there was no cold ischemia. The heart was able to support the circulation for 37 minutes with good cardiac function, except for an abnormality in the right ventricular pressure-volume loop morphology. Rosenfeldt and collegues ${ }^{22}$ evaluated the heart donated after cardiac death from a 42 year-old man who developed irreversible neurologic damage from anoxia by using their custom ex vivo perfusion system. The WIT for this heart was 32 minutes. The heart maintained adequate left ventricular pressure over 12 hours with epinephrine 
support. In our study, 4 human DCD hearts were resuscitated by our ex vivo perfusion method, except for 1 DCD heart with prolonged WIT of 174 minutes. The WIT of 4 resuscitated DCD hearts was within 40 minutes and the mean CIT was $152 \pm 55$ minutes. Our study shows that the contractile function of human DCD hearts for the first 90 minutes of reperfusion was comparable with that of BD hearts, however, DCD heart function tended to deteriorate after 2 hours of reperfusion. Although previous experiments and our results suggest that WIT in the range of 30 to 40 minutes may be acceptable, because of the potential for severe myocardial damage, it is likely that the ideal WIT for clinical adult human DCD heart transplantation would be within 20 minutes although further studies are warranted to establish the relationship between WIT and myocardial viability.

It is reasonable to expect that diastolic function in DCD donor hearts would be impaired due to several factors present in ischemic myocardium, including derangements in calcium homeostasis as a result of reduced uptake of $\mathrm{Ca}^{2+}$ into the sarcoplasmic reticulum by the sarco-/ endoplasmic reticulum $\mathrm{Ca}^{2+}$-ATPase and alterations in the function of the membrane bound sodium-calcium exchanger. ${ }^{23}$ In addition, prolonged duration of ischemia would reduce cardiac contractile function as a result of the deleterious effects of ischemia-reperfusion injury, ultimately reducing the amount of viable myocardium and leading to impaired cardiac relaxation and diastolic dysfunction. In light of these considerations, a limitation of our model arises from the inability to adequately describe diastolic dysfunction as it would pertain to DCD hearts. Our data did not show a statistical difference in diastolic function between BD and DCD hearts (dP/dt min of DCD hearts deteriorated more after 120 minutes of reperfusion than that of BD hearts). However, as shown in Figure 4, end diastolic pressure decreases with wider deviation in some BD and DCD hearts with increased balloon volume, a result that was unexpected. It is likely due to experimental considerations such as instability of the intraventricular balloon position with increasing balloon volume, especially in the diastolic phase. This suggests that the diastolic pressure measurements in this study may not reflect the exact diastolic pressures and future studies of diastolic dysfunction in DCD hearts are warranted.

\section{Study Limitations}

This study has some limitations. First, the sample size is small, which could affect the results of the statistical analysis. Second, although cardiac function of the BD donor hearts that were evaluated in this study was nearly normal before donation, most of the BD donors were declined for clinical heart transplantation because of atherosclerotic disease in the coronary artery. This indicates that these BD hearts may not be appropriate to be considered as a control group. For the DCD donors, pretransplant cardiac evaluation such as transthoracic echocardiography or coronary angiogram was not completed because these DCD donors were not considered as clinical cardiac donors. Third, our functional evaluation was an ex vivo nonworking beating heart model and this concept is originally from the Langendorff preparation, which was not able to evaluate in vivo working biventricular cardiac function. Fourth, the donor hearts were functionally evaluated only for the acute phase (the 90-minute time window) in this study. This is a major weakness of this study. Possible reasons for early functional deterioration in this study could be impairment of coronary perfusion by the intraventricular balloon, the semisterile perfusion technique without using antibiotics, and inappropriate perfusion solution without adding a high-osmotic solution such as albumin solution. Therefore, further evaluation for longer perfusion periods is required to confirm our findings. Currently, a portable ex vivo heart perfusion system is being clinically investigated as a means to optimize the use of BD hearts. ${ }^{24}$ This system can facilitate the delivery of pharmaceutical agents that support reparative processes in ischemic myocardium and the assessment of graft function before transplantation. ${ }^{25-28}$ This may be also useful for graft evaluation for longer perfusion periods. ${ }^{22}$

\section{CONCLUSIONS}

We successfully resuscitated and measured ex vivo cardiac function in human DCD and BD donor hearts. Our study has shown that the resuscitation potential in DCD hearts was achieved when the WIT was less than 40 minutes and ex vivo contractile function for 90 minutes of reperfusion in the resuscitated human DCD hearts was comparable with that of human BD hearts. However, after 2 hours of reperfusion, the graft function for DCD donors tended to deteriorate compared with the BD hearts. Although our functional observation was only for the acute phase and further evaluation is necessary to confirm our findings, our results indicate that human DCD hearts within optimal warm ischemia might be another donor source for adult cardiac transplantation.

The authors thank James E. Anderson, transplant coordinator at UWHC-OPO for assistance with DCD donor data collection.

\section{References}

1. Barnard CN. The operation. A human cardiac transplant: an interim report of
a successful operation performed at Groote Schuur Hospital, Cape Town.
S Afr Med J. 1967;41:1271-4.
2. Boucek MM, Mashburn C, Dunn SM, Frizell R, Edwards L, Pietra B, et al., Den-
ver Children's Pediatric Heart Transplant Team. Pediatric heart transplantation
after declaration of cardiocirculatory death. N Engl J Med. 2008;359:709-14.
3. Cooper JT, Chin LT, Krieger NR, Fernandez LA, Foley DP, Becker YT, et al.
Donation after cardiac death: the University of Wisconsin experience with renal
transplantation. Am J Transplant. 2004;4:1490-4. 
4. Foley DP, Fernandez LA, Leverson G, Chin LT, Krieger N, Cooper JT, et al. Donation after cardiac death: the University of Wisconsin experience with liver transplantation. Ann Surg. 2005;242:724-31.

5. Kootstra G, Daemen JH, Oomen AP. Categories of non-heart-beating donors. Transplant Proc. 1995;27:2893-4.

6. Shirakura R, Matsuda H, Nakano S, Nakata S, Kaneko M, Takami H, et al. Myocardial energy metabolism in asphyxiated canine hearts preserved for 24 hours. Transplantation. 1992;53:1215-8.

7. Gundry SR, Fukushima N, Eke CC, Hill AC, Zuppan C, Bailey LL. Successful survival of primates receiving transplantation with "dead," nonbeating donor hearts. J Thorac Cardiovasc Surg. 1995;109:1097-110.

8. Kotani Y, Ishino K, Osaki S, Honjo O, Suezawa T, Kanki K, et al. Efficacy of MCI-186, a free-radical scavenger and antioxidant, for resuscitation of nonbeating donor hearts. J Thorac Cardiovasc Surg. 2007;133:1626-32.

9. Osaki S, Ishino K, Kotani Y, Honjo O, Suezawa T, Kanki K, et al. Resuscitation of non-beating donor hearts using continuous myocardial perfusion: the importance of controlled initial reperfusion. Ann Thorac Surg. 2006; $81: 2167-71$.

10. D'Alessandro AM, Fernandez LA, Chin LT, Shames BD, Turgeon NA, Scott DL, et al. Donation after cardiac death: the University of Wisconsin experience. Ann Transplant. 2004;9:68-71.

11. Herdman R, Potts J, Institute of Medicine. Non Heart Beating Organ Transplantation: Medical and Ethical Issues in Procurement. Washington, DC: National Academy Press; 1997.

12. Abt PL, Fisher CA, Singhal AK. Donation after cardiac death in the UW: history and use. J Am Coll Surg. 2006;203:208-25.

13. Bernat JL, Capron AM, Bleck TP, Blosser S, Bratton SL, Childress JF, et al. The circulatory-respiratory determination of death in organ donation. Crit Care Med. 2010;38:963-70.

14. Gomez L, Li B, Mewton N, Sanchez I, Piot C, Elbaz M, et al. Inhibition of mitochondrial permeability transition pore opening: translation to patients. Cardiovasc Res. 2009;83:226-33.

15. Murphy E, Steenbergen C. Mechanisms underlying acute protection from cardiac ischemia-reperfusion injury. Physiol Rev. 2008;88:581-609.

16. Cooper DK. The donor heart. The present position with regard to resuscitation, storage, and assessment of viability. J Surg Res. 1976;21:363-81.

17. Osaki S, Ishino K, Kotani Y, Honjo O, Suezawa T, Kohmoto T, et al. Circulatory load during hypoxia impairs post-transplant myocardial functional recovery in donation after cardiac death. J Heart Lung Transplant. 2009;28: 266-72.

18. Jennings RB, Ganote CE. Structural changes in myocardium during acute ischemia. Circ Res. 1974;35(Suppl 3):156-72.

19. Barnard CN. Human cardiac transplantation. Am J Cardiol. 1968;22:584-96.

20. Magovern JA, Pae WE Jr, Miller CA, Waldhausen JA. The mature and immature heart: response to normothermic ischemia. J Surg Res. 1989;46:366-9.

21. Ali A, White P, Dhital K, Ryan M, Tsui S, Large S. Cardiac recovery in a human non-heart-beating donor after extracorporeal perfusion: source for human heart donation? J Heart Lung Transplant. 2009;28:290-3.

22. Rosenfeldt F, Ou R, Woodard J, Esmore D, Marasco S. Twelve-hour reanimation of a human heart following donation after circulatory death. Heart Lung Circ. 2014;23:88-90.

23. Kass DA, Bronzwaer JG, Paulus WJ. What mechanisms underlie diastolic dysfunction in heart failure? Circ Res. 2004;94:1533-42.

24. Randomized Study of Organ Care System Cardiac for Preservation of Donated Hearts for Eventual Transplantation (PROCEEDII). Available at: http:// clinicaltrials.gov/show/NCT00855712. Accessed April 1, 2014.

25. Rosenbaum DH, Peltz M, DiMaio JM, Meyer DM, Wait MA, Merritt ME, et al. Perfusion preservation versus static preservation for cardiac transplantation: effects on myocardial function and metabolism. J Heart Lung Transplant. 2008;27:93-9.
26. Collins MJ, Moainie SL, Griffith BP, Poston RS. Preserving and evaluating hearts with ex vivo machine perfusion: an avenue to improve early graft performance and expand the donor pool. Eur J Cardiothorac Surg. 2008;34:318-25.

27. Repse S, Pepe S, Anderson J, McLean C, Rosenfeldt FL. Cardiac reanimation for donor heart transplantation after cardiocirculatory death. J Heart Lung Transplant. 2010;29:747-55.

28. White CW, Ali A, Hasanally D, Xiang B, Li Y, Mundt P, et al. A cardioprotective preservation strategy employing ex vivo heart perfusion facilitates successful transplant of donor hearts after cardiocirculatory death. J Heart Lung Transplant. 2013;32:734-43.

\section{Discussion}

Dr Richard-Tien V. Ha (Stanford, Calif). Dr Osaki, thank you for providing me the manuscript ahead of time and also congratulations on the research that you have done. I think this is something that we will be talking about more in the future.

As you know, your laboratory, as well as other laboratories, have looked at animal hearts in the DCD condition. And although those results have been promising, there are a lot of hurdles to overcome. I listened with interest to your presentation and read your manuscript. And I think what stands out is that you used human hearts really for the first time in a DCD condition and used an ex vivo apparatus to try to recondition or look at their function.

The other thing that was very interesting about your paper was the fact that you chose to use a functional, or at least start to use a functional assessment rather than just biochemical assessments for the hearts, which is something novel as well.

To that end I have 2 questions for you.

The first question relates to the functional assessment. And you mentioned that it was a nonworking heart model, but in the future do you plan to assess a working heart model where you vary the preload and the afterload and challenge the heart in that condition?

The second question is: the studies that have been done by Ali and other laboratories in animals have looked at and discussed that the RV is actually a key component in the failure of these DCD hearts. Did you observe the RV at all during this time-understandably it's in a Langendorff model—or are you planning to do that in the future?

Dr Osaki. Thank you very much, Dr Ha. It's a very good comment.

For the first question, actually we didn't change the afterload but we changed the preload by the balloon volume. Yes, absolutely, in the future, we need to do an in vivo setting as a nice functional variation.

And also the second question, in this study we didn't look at the right heart function; as we know, there is 1 case report for biventricular functional evaluation for the human DCD heart, and that's a great study. Yes, absolutely, we are planning to do biventricular function analysis. 